\title{
Wenn im Herbst die Kastanien blühen
}

\author{
Hilke Steinecke
}

\begin{abstract}
Some horse chestnut trees (Aesculus) produce new leaves and flowers in autumn. This is related to stress caused by chestnust leaf miners (Cameraria ohridella) and dry and hot summers, which leads to loss of leaves in late summer. These emergency shoots can be interpreted as attempts to compensate the loss of photosynthesis.
\end{abstract}

\section{Zusammenfassung}

Im Herbst treiben manche Rosskastanien (Aesculus) Blätter und Blüten aus. Dies ist eine Reaktion auf Stress. Kastanien-Miniermotten (Cameraria ohridella) sowie trocken-heiße Sommer bewirken, dass Kastanien ihr Laub bereits im Spätsommer verlieren. Durch diese auch beblätterten Nottriebe versucht der Baum, Photosyntheseverluste auszugleichen.

Jedes Jahr überrascht es immer wieder, wenn im September und Oktober Rosskastanien frische Blüten und Blätter austreiben. Anfragen nach den Gründen dafür stehen dann auf der Tagesordnung. Auch in Frankfurt ist dieses Phänomen nicht zu übersehen. Der Palmengarten ist von verkehrsreichen Straßen wie der Bockenheimer Landstraße und der Miquelallee umgeben. An diesen Straßen sind weiß blühende Gewöhnliche Rosskastanien (Aesculus hippocastanum) sowie Fleischrote Rosskastanien (Aesculus carnea) als Straßenbäume gepflanzt. Diese zeigen jedes Jahr frische Blätter und Blüten, wenn das diesjährige Laub bereits braun ist und reife Früchte an den Bäumen hängen. Ende

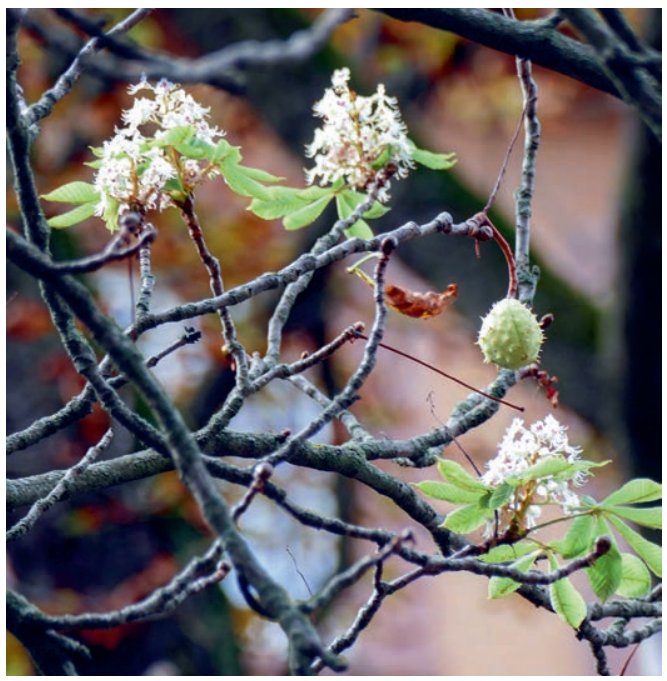

Abb. 1: Gleichzeitig blühende und fruchtende Rosskastanie (Aesculus hippocastanum) an der Bockenheimer Landstraße in Frankfurt. (Foto: H. STEinecke)
September 2020 blühten einige Bäume vielleicht sogar noch stärker als sonst.

Kastanien leiden während des Sommers aus verschiedenen Gründen unter Stress. Zum einen sind es die Kastanien-Miniermotten (Cameraria ohridella), deren Larven das innere Blattgewebe fressen, so dass die Blätter früh welken und mitunter bereits im August abgeworfen werden. Zum anderen verursachen Standorte an vielbefahrenen Straßen mit starker Versiegelung der Oberfläche, geringer Wasserzufuhr sowie einer hohen Abgasbelastung nicht unerheblichen Stress für die Bäume. Hinzu kommt, dass Rosskastanien ursprünglich vom Bal-

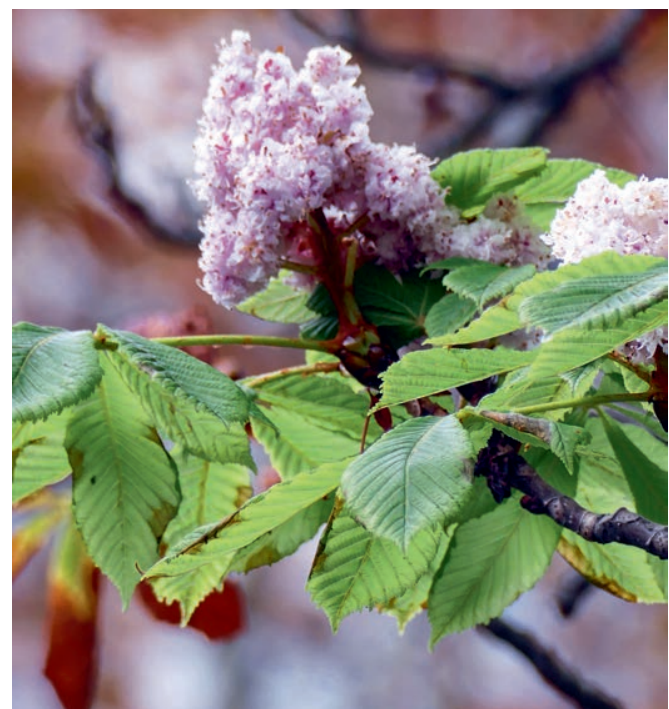

Abb. 2: Etwas gedrungener Blütenstand im September von Aesculus hippocastanum. (Foto: H. STEInecke) 


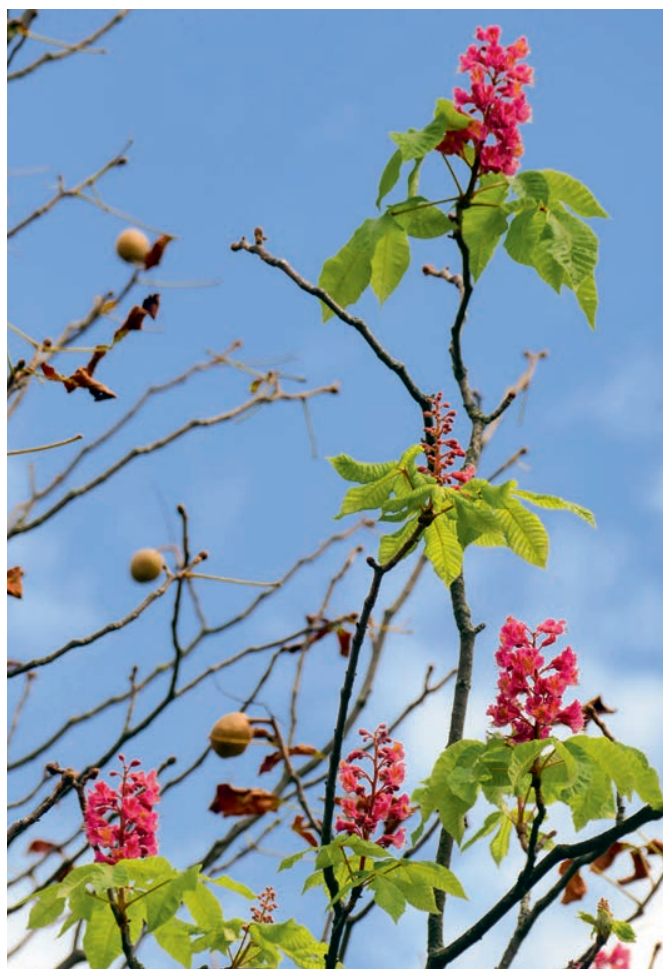

Abb. 3: Gleichzeitig Blüten und Früchte an Aesculus carnea an der Miquelallee in Frankfurt. (Foto: H. Steinecke)

kan stammen, wo sie bevorzugt an den Hängen kühler, feuchter Schluchten wachsen. Dort herrschen völlig andere Standortbedingungen vor als an städtischen Straßenrändern. Seit einigen Jahren sorgen heiße, trockene Sommer für zusätzlichen Stress für viele Baumarten.

Die meisten Kastanien sehen also bereits im Spätsommer stressbedingt sehr schlecht aus und werfen ihr Laub ab. Die volle Jahres-Photosyntheseleistung kann dann nicht erfüllt werden. Durch die Entfaltung neuer photosynthetisch aktiver Blätter versucht der Baum, dieses Defizit auszugleichen. Gefördert werden diese Nottriebe vor allem dann, wenn es nach einem trocken-heißen Sommer im Herbst endlich einmal regnet. Temperatur- und Lichtverhältnisse gleichen denen im Frühling, derjenigen Zeit, in der die Kastanien normalerweise austreiben.

Die Triebe haben gegen Ende der Vegetationsperiode allerdings nicht mehr die Gelegenheit,



Abb. 4: Fleischrote Kastanien an der Miquelallee blühen und fruchten im September. (Foto: H. STEINecke)

richtig auszuhärten, so dass sie im folgenden Winter absterben. Die im Herbst ausgetriebenen Knospen fehlen dann im nächsten Frühjahr, weshalb der Baum mit diesen Nottrieben keinen „Gewinn einfahren“ kann. Mehrjährige Beobachtungen lassen aber vermuten, dass Kastanien das regelmäßige Austreiben eines Teils ihrer Knospen im Herbst relativ gut überstehen und im darauffolgenden Frühling wieder in voller neuer Blüten- und Laubpracht erscheinen.

\section{Internetseiten}

https://www.krautundrueben.de/kastanienbluete-herbst https://www.berliner-woche.de/lichterfelde/c-umwelt/kastanie-traegt-fruechte-und-blueht-zugleich_a180614

\section{Anschrift der Autorin:}

Dr. Hilke Steinecke, Palmengarten Frankfurt, Siesmayerstraße 61, 60323 Frankfurt, E-Mail: hilke.steinecke@stadt-frankfurt.de 